Trascender, Contabilidad y Gestión Núm. 13 (enero - abril del 2020).

Universidad de Sonora. Departamento de Contabilidad.

ISSN: 2448-6388. Reserva de Derechos 04-2015-04172070800-203.

\title{
Políticas públicas para la prevención y atención de conductas atípicas en menores: una reflexión desde el contexto internacional y mexicano
}

Public policies for the prevention and care of atypical behaviors in minors: a reflection from the international and Mexican context

Miguel Ángel Vega Campos ${ }^{1}$

Recibido: 14 de septiembre de 2019.

Aceptado: 19 de noviembre de 2019.

DOI: https://doi.org/10.36791/tcg.v13i0.79

JEL: I18. Política pública; Regulación.

\section{Resumen}

Existen diferentes factores y circunstancias que provocan en los menores y jóvenes conductas atípicas que ponen en riesgo su convivencia regular dentro de la sociedad. Por esta razón, la participación del gobierno -independientemente del ámbito de administración pública de que se trate: federal, estatal o municipal- resulta trascendental, en cuanto al diseño e implementación de políticas públicas que permitan prevenir y atender conductas atípicas que presentan los menores y los jóvenes, de tal forma que esta cuestión no derive en un problema de mayores dimensiones, cuyos efectos pongan en riesgo la convivencia adecuada de los ciudadanos. Sabiendo que la problemática antes aludida no es exclusiva de México, este trabajo tuvo como propósito realizar una revisión y análisis de la literatura, tanto en el contexto mexicano como a nivel internacional, de publicaciones académicas o de investigación, en las que se reseñan acciones, programas o políticas públicas, implementadas para la prevención y atención de conductas atípicas en menores y jóvenes; de tal forma que dicha revisión y análisis permitiera al autor de este ensayo, esgrimir algunas recomendaciones o conclusiones sobre la temática abordada. Derivado de la revisión de la literatura, tanto en el contexto internacional como en el mexicano, en torno a políticas públicas para la prevención y atención de conductas atípicas en menores o jóvenes, se puede señalar que se tienen suficientes evidencias que pueden aprovecharse como referencia o punto de partida, para abordar esta problemática que no es ajena a México.

\footnotetext{
${ }^{1}$ Miguel Ángel Vega Campos. Licenciado en Administración Pública. Maestro en Administración. Doctor en Administración. Profesor investigador de tiempo completo, y Coordinador de la Maestría en Dirección Empresarial del Centro de Investigación y Estudios de Posgrado de la Facultad de Contaduría y Administración de la Universidad Autónoma de San Luis Potosí, México. Líder del Cuerpo Académico consolidado Innovación y Gestión en las Organizaciones. Miembro de la Red Administración y Gestión en las Organizaciones. Correo: miguel.vega@uaslp.mx. ORCID: 0000-001-5576-863x
} 
Palabras clave: políticas, públicas, conductas, atípicas, menores.

\begin{abstract}
There are different factors and circumstances that provoke atypical behaviors in minors and young people that put their regular coexistence within society at risk. For this reason, the participation of the government regardless of the sphere of public administration in question: federal, state or municipal- is transcendental, in terms of the design and implementation of public policies that allow preventing and addressing atypical behaviors presented by minors and young people, so that this issue does not lead to a larger problem, the effects of which jeopardize the proper coexistence of citizens. Knowing that the aforementioned problem is not exclusive to Mexico, this work aimed to carry out a review and analysis of the literature, both in the Mexican context and at the international level, of academic or research publications, in which actions are outlined, programs or public policies, implemented for the prevention and care of atypical behaviors in minors and youth; in such a way that said review and analysis allowed the author of this essay to use some recommendations or conclusions on the topic addressed. Derived from the literature review, both in the international and in the Mexican context, regarding public policies for the prevention and care of atypical behaviors in minors or young people, it can be pointed out that there is sufficient evidence that can be used as a reference or starting point, to address this problem that is not foreign to Mexico.
\end{abstract}

Keywords: public, policies, behaviors, atypical, minors.

\section{Introducción}

Aun cuando pareciera que la frase "los menores y los jóvenes son el futuro y hay que cuidarles y educarles”, suena demasiado trillada, la expresión sigue siendo vigente en cualquier sociedad y circunstancia; por lo que esta cuestión debe ser un foco rojo que ponga en alerta el funcionamiento de la administración pública independientemente del orden de gobierno de que se trate: federal, estatal o municipal-, en cuanto al diseño e implementación de políticas públicas que procuren encausar de forma adecuada, el comportamiento de los menores y los jóvenes, dentro de una sociedad que cada vez está más fragmentada y descompuesta. En definitiva, el gobierno tiene la obligación de proporcionarles a los menores y jóvenes un camino de futuro en las mejores condiciones posibles.

Son diferentes factores y circunstancias los que pueden provocar en los menores y jóvenes, conductas atípicas que ponen en riesgo su convivencia regular dentro de la sociedad. En este sentido, autores como Nieto-Morales (2012) exponen que la familia es la unidad básica de socialización y se constituye como el pilar fundamental para que los menores y los jóvenes adquieran valores que les permitan un futuro normalizado; sin embargo, la familia sola no puede educar y es la escuela que además contribuye a esta socialización, la que facilita la integración, siendo muy importante también el aprendizaje social. Por lo tanto -como bien lo señala Nieto-Morales (2012:17)-, "todos estos ingredientes son los que permiten que los menores y jóvenes puedan tener una vida personal y social normalizada”.

Sin embargo, "con los cambios sociales, la estructura familiar ha sufrido importantes modificaciones $\mathrm{y}$ en muchos casos pérdida de valores fundamentales para la 
educación de los hijos” (Nieto-Morales, 2012, p, 17); lo cual -sigue exponiendo el mismo autor- ha propiciado que los menores y jóvenes se vean involucrados en actos de violencia y delincuencia, que no se constituyen como hechos sociales aislados, ya que se encuentran inmersos en contextos sociales mucho más complejos, imbricados en estructuras sociales, económicas, legales, políticas, etc.

Ante este panorama, la participación del gobierno resulta trascendental, en cuanto al diseño e implementación de políticas públicas que permitan prevenir y atender conductas atípicas que presentan los menores y los jóvenes, de tal forma que esta cuestión no llegue a convertirse en un problema de mayores dimensiones, cuyos efectos pongan en riesgo la convivencia adecuada de la sociedad en general.

Sabiendo que la problemática antes aludida no es exclusiva de México, este trabajo tuvo como propósito realizar una revisión y análisis de la literatura, tanto en el contexto mexicano como a nivel internacional, de publicaciones académicas o de investigación, en las que se reseñan acciones, programas o políticas públicas, implementadas para la prevención y atención de conductas atípicas en menores y jóvenes; de tal forma que dicha revisión y análisis permitiera al autor de este ensayo, esgrimir algunas recomendaciones o conclusiones sobre la temática aquí abordada.

\section{Desarrollo del documento}

\section{Conceptualización de la violencia juvenil, su origen y esquemas para su prevención}

En términos de algunos autores, la violencia es un problema que ha estado asociado a la juventud a lo largo del tiempo. Desde una perspectiva biológica, otros autores exponen:

Que la carga genética que se ha desarrollado a través de la evolución explica por qué los hombres jóvenes son más propensos a ser violentos que las mujeres o los viejos, la causa es que nuestros ancestros jóvenes violentos tenían más posibilidad de sobrevivir y reproducirse que el resto (Potts \& Hayden, 2010, citados por Mancha \& Ayala, 2018:175).

La violencia y la delincuencia en jóvenes también se explica a partir de problemas tanto psicológicos como biológicos (INEGI, 2015, citado por Mancha \& Ayala, 2018). Es así que la prevención del delito se puede abordar desde varios enfoques. Por un lado, otros autores señalan que:

El enfoque del desarrollo se basa en la identificación de los factores de riesgo y factores de protección que influyen en que un individuo cometa o no un acto violento o delito. Las teorías en que se fundamenta el enfoque del desarrollo buscan responder las preguntas: ¿por qué una persona se convierte en delincuente?, y ¿por qué una persona decide cometer o dejar de cometer delitos? (Mancha \& Ayala, 2018:175-176).

Por otro lado, siguiendo con Mancha y Ayala (2018:176), estos autores mencionan que:

La teoría de la interacción, por su parte, considera que la violencia y la delincuencia se aprenden y refuerzan por la interacción del individuo con su entorno y sus lazos sociales (ya sean familiares o de amistad). Mientras que la teoría de los rangos de edad considera que factores estructurales como el nivel de ingresos, familias disfuncionales o con alta cohesión, desarrollo de la ciudad, entre otros, así como la fuerza de los lazos sociales, están asociados a las probabilidades de que un individuo exhiba un comportamiento violento o delictivo (INEGI, 2015:176). 
Por lo tanto, expone Fagan que:

Según estas teorías, los niños y jóvenes aprenden el comportamiento (positivo o negativo) con la interacción con otras personas, y los padres son particularmente importantes para influenciar a sus hijos; así, padres que muestran actitudes favorables hacia comportamientos desviados o que no corrigen esta clase de comportamiento de sus hijos incrementan la probabilidad de que ellos vean la violencia y las actividades delincuenciales como aceptables para alcanzar ciertos resultados, en especial, cuando perciben más beneficios que costos de dichos actos (Fagan, 2013, citado por Mancha \& Ayala, 2018:176).

Sobre el mismo orden de ideas, se indica que:

La violencia tiene orígenes, causas, instrumentos y mecanismos específicos. No es una expresión natural, sino un fenómeno estrictamente humano. A medida que transcurre el tiempo, las formas que la violencia ha adquirido en el acontecer histórico se ha diversificado más, ensanchando al mismo tiempo el campo de análisis científico y de reflexión filosófica (Pérez, 2016:46).

Por lo que se refiere a la violencia juvenil, Pérez (2016) señala que no existe un consenso conceptual de los estudiosos del tema, "para tal caso, tiende a emplearse lo relativo a la delincuencia juvenil como la violación de la ley comprendida entre los 10 y 29 años” (p. 47). En este sentido, indica el mismo autor que:

La Organización Mundial de la Salud (OMS) define como violencia juvenil al involucramiento de jóvenes (entre 10 y 29 años), como víctimas o agresores, en el uso intencional de la fuerza física o el poder, como amenaza o acción efectiva, contra sí mismo, contra otra persona o en contra de un grupo o comunidad, ya sea que produzca o tenga una alta probabilidad de producir una lesión (daño), la muerte, daño psicológico, mal desarrollo o la privación de la(s) persona(s) agredida(s) (Pérez, 2016:47).

Autores como Moro (2006, citado por Pérez, 2016:47) señalan que la violencia juvenil puede distinguirse en dos planos distintos: por un lado el estructural, que expone las condiciones materiales en que los jóvenes viven y que hace visible la desigualdad y la exclusión. Por otra parte, se habla también de violencia activa, “en la cual los jóvenes pasan a ser victimarios y que en su aspecto disruptivo hace referencia a 'manifestaciones espamódicas, o bien de cierta continuidad en algunas geografías, pero siempre manifestándose como actos de desbordaje que despliegan, a veces, niveles de brutalidad en el límite de lo imaginable’”.

Se hace referencia a la violencia estructural, al hablar de jóvenes como receptores de la violencia que ejerce la sociedad sobre ellos; la cual puede ser de cuatro tipos; la económica, la política, la sociocultural y la urbana (Pérez, 2016). Siguiendo con el mismo Pérez (2016), éste explica que también existe una violencia a nivel institucional, en la que el joven es objeto en este nivel, y que puede ser ejercitada desde la policía, la escuela, las instituciones hospitalarias, y por las políticas y normas diseñadas para contener el comportamiento de lo que la sociedad adultocéntrica considera "inadecuado".

Factores de riesgo asociados a conductas violentas en menores y jóvenes de México

Existen dos tipos de factores de riesgo predominantes que están correlacionados a los actos violentos de gritar, golpear objetos, golpear personas, portar armas o haber sido arrestado, que cometen los jóvenes: 
1) factores relacionados con adicciones (drogas o alcohol) y 2) violencia dentro del hogar o en el entorno cercano (violencia genera violencia). Actos de violencia más graves (portar armas o haber sido arrestado) presentan una mayor correlación con factores relacionados a las adicciones, mientras que actos violentos menos graves (gritar, golpear objetos o gritar personas) presentan correlación tanto con factores relacionados con las adicciones como con factores relacionados con la violencia en el entorno (Mancha \& Ayala, 2018:171).

Siguiendo con Mancha y Ayala (2018), estos autores mencionan que los resultados de estudios empíricos han encontrado consistentemente que los niños y jóvenes expuestos a la violencia, ya sean como testigos o como víctimas, tienen un mayor riesgo de desarrollar patrones de comportamiento agresivo y violento. En este sentido, otros autores exponen que:

Específicamente, se ha encontrado que factores en el ambiente familiar como abuso de drogas o alcohol por parte de los padres, ambientes inestables, violencia doméstica, disciplina parental muy estricta, así como vecindarios violentos son factores de riesgo para detonar el comportamiento violento en los jóvenes (Fehon, 2007; Soto \& Trucco, 2015, citados por Mancha \& Ayala, 2018:177).

Por lo que hace al caso de México, "los estudios y las estadísticas de medición e identificación de factores de riesgo de personas violentas, de delincuentes, la prevención y victimización son muy limitados y recientes” (Cortez, 2015; González, 2014, ambos citados por Mancha \& Ayala, 2018:178). Es así que:

Los estudios de esta problemática desde un enfoque económico están en su etapa inicial y los estudios existentes se centran principalmente en tres temas: "la identificación de los delitos más comunes, los espacios geográficos con mayores índices delictivos, así como la descripción de las características sociodemográficas de las víctimas” (Cortez, 2015, citado por Mancha \& Ayala, 2018:178).

Como se puede observar, la problemática ha sido abordada de manera muy superficial y con mayor concentración o enfoque en la victimización, que en la delincuencia o criminalidad. Al respecto:

Son casi inexistentes los estudios que aborden los factores que detonan la violencia $y$, en particular, la violencia en los jóvenes, los pocos estudios en este tema son de corte cualitativo y estudios de caso. La falta de datos, [...], puede ser uno de los responsables de que el fenómeno esté tan poco explorado (Mancha \& Ayala, 2018:178).

Conceptualización de política pública y su importancia para la prevención y atención de conductas atípicas en menores y jóvenes

No obstante que existen diversas definiciones de políticas públicas, se considera que la proporcionada por Velásquez (2009) resulta pertinente en este trabajo, en virtud de que dicho concepto engloba los siguientes cuatro elementos:

1) Proceso integrador de decisiones, acciones, inacciones, acuerdos e instrumentos;

2) Proceso adelantado por autoridades públicas con la participación eventual de los particulares;

3) Proceso encaminado a prevenir o solucionar una situación definida como problemática; y

4) La política pública hace parte de un ambiente determinado del cual se nutre $\mathrm{y}$ al cual pretende modificar o mantener. 
Es así que la política pública se define como:

Un proceso integrador de decisiones, acciones, inacciones, acuerdos e instrumentos, adelantado por autoridades públicas con la participación eventual de los particulares, y encaminado a solucionar o prevenir una situación definida como problemática. La política pública hace parte de un ambiente determinado del cual se nutre y al cual pretende modificar o mantener (Velásquez, 2009:156).

Para el caso específico que nos ocupa en este documento, resulta necesario hablar de políticas públicas de juventud, lo cual -como bien lo expone Pérez (2016)-, se trata de las políticas públicas orientadas a los jóvenes en la agenda de las actividades estatales. En este orden de ideas, Dina Krauskopf (2011; citada por Pérez, 2016:9-10) conceptualiza a las políticas públicas de juventud "como el principal instrumento de referencia de un sistema de acciones dirigidas a la juventud”.

Sobre el mismo tema, se indica que:

El desarrollo de políticas de juventud y la investigación asociada a ella, resulta de especial complejidad pues requiere un continuo grado de conocimientos sobre la manera en la que las juventudes van cambiando y cuáles son sus características demográficas, culturales, sociales, de género, etnográficas, económicas, etcétera (Pérez, 2016:10).

Los autores coinciden en señalar que el trato dado por las políticas públicas a los jóvenes ha sido preponderantemente asistencial y sectorial (Pérez, 2016); ya que se ha visto a los jóvenes como sujetos pasivos y receptores de la ayuda del Estado en los más diversos ámbitos: educación, salud, empleo, por ejemplo; y sectorial porque dichas intervenciones han estado agrupadas dentro de las políticas públicas orientadas al grueso de la población y no de manera específica para atender las necesidades de este sector.

Algunos autores como Ernesto Rodríguez (1999, citado por Pérez, 2016:13-18), proponen cuatro modelos de políticas públicas, para la atención de los menores y los jóvenes: 1) Educación y tiempo libre con jóvenes integrados y crecimiento económico. Este modelo de política de juventud se originó durante las décadas de mayor crecimiento económico reciente (1950-1980), es decir, en la época del boom económico que siguió a la posguerra y se concentró por una parte, en otorgar y extender los servicios educativos, y por otra, en atender el tiempo libre de los jóvenes; 2) Control social de jóvenes movilizados en un marco de crecientes tensiones. Este modelo de políticas de juventud estuvo asociado a la movilización juvenil organizada que propició la incorporación masiva de los jóvenes al sistema educativo. Dichas políticas, que han persistido con nuevas hibridaciones hasta nuestros días, se caracterizaron por el desarrollo de medidas de control social que fueron tradicionalmente desempeñadas por ministerios de gobernación o del interior; 3) Enfrentamiento de la pobreza y el delito en un marco de crisis generalizada. El desarrollo de este modelo a partir de 1980 estuvo vinculado con el fin de la era de crecimiento sostenido que había visto la economía internacional desde inicios de la década de 1950. Es bien sabido que en esta época comenzó a ser implementado el llamado modelo neoliberal en América Latina. Por otra parte "el monetarismo", doctrina económica en boga desde el llamado Consenso de Washington, comenzó a orientar los ejes de la política económica al plantear mayor austeridad en el gasto social, la privatización del sector público y la desregulación comercial; y 4) Inversión en capital humano en el marco de la transformación educativa. Este último modelo de 
política de juventud, está relacionado con la intención que desde inicios de los años noventa se propuso insertar laboralmente a los jóvenes dentro del marco del "desarrollo de un capital humano".

Siguiendo con el tema de las políticas públicas para menores y jóvenes, diversos autores distinguen dos tipos: las punitivas y las preventivas. En este sentido, Guemureman (2011; citada por Pérez, 2016) señala que:

\section{Las orientaciones punitivas recurren} invariablemente al sistema penal, la coerción y el control, en lugar de gobernar a través de políticas públicas que reviertan o mejoren al menos, las condiciones de vida de los grupos más vulnerables, para así promover 'soluciones' a mediano y largo plazo (Pérez, 2016:19).

Por otra parte, se define a una política pública preventiva como aquella que:

[...] establece mecanismos que impiden que la violencia alimente las dinámicas sociales que llevan al siguiente nivel del proceso de agrupación juvenil delictiva. En otros términos, ello implica el desarrollo de políticas públicas capaces de articular estrategias de intervención que consisten en la prevención primaria para los niveles más bajos de la ruta de la violencia (familia, grupos juveniles), prevención secundaria para grupos como las barras juveniles [...] (Cruz \& Carranza, sin fecha, citados por Pérez, 2016:19-20).

\section{Políticas públicas para la prevención y atención de conductas atípicas en menores: una revisión de la literatura}

\section{Contexto internacional}

En un trabajo titulado "La estrategia europea de la prevención de la delincuencia a través del incivismo a examen”, cuyo propósito fue analizar si la estrategia política preventiva del delito se ha centrado también en la regulación de los actos incívicos; además de investigar si a nivel de la Unión Europea (UE) se ha prestado atención a la cuestión relativa a cómo las autoridades locales ha ejercido sus poderes de prevención del delito (PD) en el ámbito del desorden urbano, Anna Di Ronco (2017:16), llegó a las siguientes conclusiones:

Los resultados muestran que los actos incívicos (entendidos como la conducta o el comportamiento antisocial que genera sensación de inseguridad) se han incluido en la estrategia de la UE de PD y que dicha estrategia se ha centrado principalmente en facilitar la cooperación entre los países miembros y la Unión. Aunque las instituciones de la UE han reconocido el valor de los esfuerzos en materia de prevención de las autoridades locales y los han apoyado sustancialmente mediante la apropiación de los fondos de la UE, parece que han prestado escasa atención a la forma en que dichas autoridades han ejercido sus poderes de $P D$, en particular, respecto a su impacto en las libertades de los individuos.

[...], la reducción de la atención al ejercicio local de los poderes de seguridad pública y, sobre todo, a sus repercusiones en los derechos fundamentales de las personas puede explicarse por la limitada competencia de la UE en el ámbito de la PD (Di Ronco, 2017:16).

Por otro lado, en un documento denominado " $L o$ político y las políticas de la delincuencia juvenil: Una reflexión sobre el caso de jóvenes vinculados al Sistema de Responsabilidad Penal Adolescente. BogotáColombia", cuyo objetivo fue reflexionar en torno a los sentidos y significados del delito en jóvenes vinculados al Sistema de Responsabilidad Penal Adolescente (SRPA) en Bogotá - Colombia, Ana Guadalupe Cienfuegos (2015:14-17) concluye que: 
[...] la problemática de la delincuencia juvenil no es solo un asunto técnico que debe resolverse desde la intervención punitiva y/o asistencial, es también, al parecer, un asunto político que implica la comprensión de la naturaleza de identidades colectivas en contextos de exclusión social, y el reconocimientos de los jóvenes vinculados al delito como actores con necesidades sociopolíticas desatendidas.

[...] lo ético en estos jóvenes, [...] no es un objeto a ser transformado desde discursos externos, pues forma parte de un entramado de sentidos y significados que se construyen en un territorio donde la noción de justicia, poder y ética atraviesa por la dimensión política de los jóvenes y los grupos como uno de los aspectos articulados al sentido que tiene la delincuencia para muchos jóvenes [...].

Los lineamientos de políticas públicas para la prevención y atención de la delincuencia juvenil destacan como uno de los propósitos centrales de su quehacer el 'favorecer al ejercicio ciudadano de los jóvenes que se vinculan a delitos'; no obstante, la estigmatización social hacia estos adolescentes es cada vez mayor en los medios de comunicación, la comunidad en general y desde el propio Estado.

Las políticas públicas para hacer frente al fenómeno parecen suponer que las leyes en sí mismas transforman imaginarios sociales y que, por tanto, la desestigmatización y despatologización de los adolescentes vinculados al delito no es asunto de estas. No hay pedagogía de las leyes y políticas de prevención de la delincuencia juvenil, además, las políticas para afrontar el fenómeno escasamente contemplan estrategias pedagógicas y mediáticas orientadas a la desestigmatización social de los adolescentes que se vinculan al delito; en su lugar, parecen haber olas intermitentes de estigmatización y sensacionalismo en torno a la delincuencia juvenil desde los medios de comunicación y diversos sectores políticos y sociales. ¿Cómo pretenden entonces las políticas aportar al desarrollo de identidades sociales y colectivas positivas de los jóvenes?, ¿es posible generar transformaciones éticas desde discursos y valores externos?

La desestigmatización social y la despatologización de los jóvenes en riesgo de vincularse al delito y de quienes ya lo han hecho son procesos centrales, que desde las políticas públicas podrían aportar a la dimensión éticopolítica de los jóvenes y los grupos juveniles; sin embargo, no son procesos en los que realmente dichas políticas públicas trabajen. La visión del 'delincuente' reforzada en imaginarios colectivos de rechazo y el punitivismo en la sociedad deja a los adolescentes en un limbo entre adolescentes vulnerables y adolescentes responsables que en la práctica parecen no ser más que un delincuente adulto común. Esta realidad reproduce en los jóvenes los sentidos y significados asociados a la dimensión ético-política que refuerzan, reproducen y mantienen el delito (Cienfuegos, 2015:14-17).

Siguiendo con la revisión de la literatura, en un trabajo titulado "Adolescentes, jóvenes y violencia en Montevideo: Una aproximación descriptiva”, cuyo propósito fue medir las formas, las frecuencias y las intensidades de la violencia policial sobre adolescentes y jóvenes (de 13 a 29 años) en el departamento de Montevideo, Montesriro, Samudio, Patermain, Salamano, Zoppolo y Tomasini (2016:75-79), concluyen 
con relación al tema que aquí se trata que:

[...] la Policía dedica ingentes esfuerzos para el control por el control mismo, bajo criterios de selectividad social y territorial, sin que ello tenga ningún correlato positivo en materia de prevención, investigación y conjuración del delito. Incluso podría señalarse que ese patrón de comportamiento afecta negativamente los perfiles de percepción y evaluación de la propia ciudadanía.

Esta investigación revela que hay un volumen importante de intervenciones diarias de la Policía sobre adolescentes y jóvenes, en especial en la vía pública. Además, un porcentaje significativo de los mismos son intervenidos de forma frecuente, lo que reafirma la idea de la focalización. Muchos de esos adolescentes y jóvenes reconocen que la intervención ocurrió en el último año, lo que tal vez indique la posibilidad de alguna subestimación cuando la referencia temporal es un poco más amplia (de haber subestimación, afectaría a los jóvenes mayores de 18 años).

Las normas que regulan las relaciones entre la Policía y la ciudadanía son escasamente conocidas por los adolescentes y los jóvenes, a lo que deberíamos sumar también [...] que los propios policías trabajan sin conocimientos sólidos sobre su marco de actuación. A este desconocimiento, hay que agregar que la violencia policial apenas se denuncia: de los adolescentes y jóvenes que han recibido malos tratos de la Policía en cualquiera de las instancias analizadas, solo un 5\% realizó la denuncia. Si el Estado no se hace responsable de desarrollar líneas de formación en materia de normas básicas que regulan las relaciones entre el uso de la fuerza y la ciudadanía, y al mismo tiempo no se habilitan lugares e instancias de denuncias diferentes a las propias oficinas policiales, la violencia institucional seguirá naturalizada y el ejercicio del control estatal no podrá evitar una profunda deslegitimación.

El uso de la fuerza legítima debe ser regulado y controlado. Para eso se requieren normas que protejan los derechos y las libertades. Pero también son necesarias estrategias de transformación organizativa hacia un modelo de gestión policial orientado a la resolución de los problemas y atento a la calidad de los procesos. Si las policías continúan gobernadas por criterios reactivos de selección criminalizante y por pautas de eficacia tradicional (detenidos, procesados, encarcelados), sus formas cotidianas de funcionamiento tenderán a reproducir la violencia institucional (Montesriro, et al., 2016:75-79).

En el mismo tenor de ideas, derivado de un trabajo denominado "Influencia de las tecnologías de información y comunicación en la violencia de los niños, niñas y jóvenes en Guatemala”, Villeda (2019:64), concluye lo siguiente:

Las tecnologías de información y los medios de comunicación destacan un notable interés en torno a los efectos negativos y positivos que pueden derivar de su uso, proporcionan oportunidades nuevas y apasionantes para explorar el mundo social, pero amenaza con socavar las relaciones y comunidades humanas, especialmente para las nuevas generaciones, por lo que es necesario tener en cuenta que se debe analizar y considerar diferentes alternativas para combatir los contenidos ilícitos aplicando la legislación existente o modificando a través de iniciativas de ley donde se defina la responsabilidad civil y penal a los usuarios, 
intermediarios y proveedores de contenidos. Asimismo se deben proponer medidas para facilitar el desarrollo de tecnología que ayude a controlar el acceso y la publicación de contenidos ilícitos y dañinos a través de las Tecnologías de Información y Comunicación.

Proponer medidas preventivas, en materia de políticas sociales para contener las causas estructurales que potencian las nuevas formas de violencia en todas sus dimensiones, demostrando la importancia de trabajar con los factores de riesgo en el fenómeno de la violencia especialmente desde el uso de las TIC, para niños, niñas y adolescentes (Villeda, 2019:64).

En un trabajo de investigación desarrollado en Argentina, titulado "Reconfiguraciones de la cuestión social: Jóvenes, drogas, violencias y políticas de prevención social del delito", cuyo propósito fue analizar los sentidos desde los cuales los representantes de diversas instituciones locales y funcionarios públicos conciben a la relación entre jóvenes, consumo problemático de sustancias y violencia como una suerte de nueva cuestión social, Llovera (2017), llegó a las siguientes reflexiones:

El problema droga, entendido como construcción social (Touzé, 2014) no permanece ajeno a la construcción social de la juventud como problema, elementos centrales del modo en que son reconfiguradas la cuestión social y las políticas de control desde los discursos hegemónicos (Grassi, 2003).

[...] la juventud es cuestionada y responsabilizada ante la escasa posibilidad de realización de ciertos ideales de estabilidad, cereza y concreción perdurables (Chaves, 2012). Estos como es notorio respecto al consumo de

Miguel Ángel Vega Campos bienes materiales, presentan numerosas dificultades para ser cristalizados. Dificultades que, si bien son atribuidas a factores individuales, poseen un correlato social que podemos ligar a las condiciones socioeconómicas de la ciudad y a las desigualdades estructurales que se originan y reproducen allí: imposibilidades de acceso al espacio público y a la movilidad, deterioro de las condiciones laborales, retraimiento de la participación social.

[...], se responsabiliza a los jóvenes por el fracaso de ciertas promesas de integración social propias del proyecto moderno de sujeto: individual, autónomo, racional, realizado (Renoldi, 2012). Sin reflexionar las posibilidades e imposibilidades de concreción de los mismos en sus trayectorias de vida [...]”. "Cabría entonces preguntarse si es realmente problemática la juventud per se o si las conflictividades sociales, económicas y espaciales impactan de un modo particular en los jóvenes [...].

Ante un panorama que plantea el devenir sujeto ligado a la realización del consumo individual de diversos bienes, sería deseable pensar en correlatos estatales que permitan la interpelación de los jóvenes en tanto sujetos, desde otras aristas. Un espacio clave podría constituirlo la participación juvenil en la hechura de las políticas públicas, en las cuales sean tomados como actores reconocidos, legítimos $y$ constructores, en lugar de meros objetos o destinatarios. [...], podría explorarse cómo los procesos $y$ condiciones estructurales que atraviesan las vidas cotidianas de los jóvenes de un municipio de rango medio se imbrincan en la construcción de modulaciones subjetivas particulares (Llovera, 2017, s/p.). 
En un documento elaborado en Puerto Rico, y denominado "Prevención de la delincuencia juvenil: ¿Qué deben tener los programas para que sean efectivos?", cuyo objetivo fue identificar y describir las características, factores y elementos que deben tener los programas de prevención de delincuencia juvenil efectivos, según jóvenes que participaban en programas que pretenden prevenir la delincuencia juvenil y profesionales expertos y expertas en el tema; Negrón y García (2016:117) encontraron que:

[...], los resultados de esta investigación apoyan la creación de programas de prevención de delincuencia juvenil que atiendan a la población general, desde la niñez con la integración y colaboración de diversos sectores. Es necesario crear una política pública en Puerto Rico dirigida a prevenir la delincuencia juvenil. En términos de los factores a considerar, los/as participantes indicaron la autoestima saludable, tener control de sí mismos/as, tener buenas calificaciones, recibir apoyo de sus familiares, pertenecer a asociaciones deportivas y obtener modelaje positivo de las figuras de autoridad del país. Entre los elementos que deben incluir estos programas destacaron el apoyo familiar, el orientarles sobre formas alternas de terminar la escuela y crear asociaciones deportivas tanto en la escuela como en la comunidad donde vive el/la joven. Estas sugerencias enfatizan la necesidad de intervenir en todos los niveles y en los variados contextos dentro de los cuales ocurre la vida cotidiana de estos/as jóvenes.

Esta investigación genera información valiosa para aquellos/as interesados en la prevención de la delincuencia juvenil. Muchos de sus resultados rebasan la realidad puertorriqueña y pueden ser de utilidad en otros países. Sirven para guiar el desarrollo de programas específicos y de políticas públicas abarcadoras que deben estar motivadas por el bienestar ciudadano y por la búsqueda de un desarrollo óptimo para nuestra juventud (Negrón \& García, 2016:117).

\section{Contexto mexicano}

En un estudio titulado "Impacto del narcotráfico en jóvenes de Tamaulipas, México: drogas e inseguridad”, que tuvo por objeto conocer la experiencia de hombres y mujeres jóvenes con respecto al narcotráfico y su impacto en el consumo de drogas, la participación en grupos delictivos y la inseguridad, Gómez y Almanza (2016:445), señalan que:

Los resultados indican que la violencia $e$ inseguridad generada por el narcotráfico ha motivado a las y los jóvenes a alejarse del consumo de drogas ilegales, u optar por drogas de fácil acceso, como estrategia de afrontamiento frente a los constantes intentos realizados por integrantes del crimen organizado para reclutar a los jóvenes (Gómez \& Almanza, 2016:445).

Por otra parte, en un trabajo denominado "Prevención del delito en adolescentes en el Estado de México. La idea de riesgo y el sistema de preceptorías”, cuyo propósito fue analizar las estrategias de prevención del delito de dichas preceptorías juveniles en el Estado de México, elaborando un diagnóstico del diseño y funcionamiento de las mismas, así como un breve abordaje de las condiciones de su operación; Niño, Osorio y González (2017:288-290), concluyen que:

Los esfuerzos institucionales de prevención del delito de adolescentes en el estado de México se caracterizan por ser resultado de un marco legal garantista vigente desde el año 2006; sin embargo, la realidad operativa de las preceptorías nos indica que subsisten posturas 
que forman parte del modelo anterior, en los que se omite el diagnóstico estructural de la vulnerabilidad como factor para explicar la conducta delictiva o antisocial. Si bien se reconoce una noción de riesgo, viene acompañada de otro concepto que es poco claro y que eventualmente puede reflejar un cúmulo de prejuicios que se traduzcan en obstáculos para la atención en los términos garantistas: la contaminación.

[...], hay que señalar que sobresale el voluntarismo individualista dentro de las preceptorías para explicar casos de éxito o fracaso en la reintegración, y se deja en un segundo plano la necesidad de modificar el entorno del menor para mejorar su experiencia de vida en lo general. Ello puede entenderse como resultado de varias problemáticas, aunque una posible explicación es la aceptación implícita de discursos psicológicos hegemónicos, que llevan a pensar que el problema de los jóvenes con conductas antisociales es resultado de fallas particulares de familias, por lo que su solución depende igualmente de las disposiciones individuales de las familias y los jóvenes. Aunque también puede deberse a los recursos limitados con que cuentan las preceptorías, lo cual las lleva a desconsiderar acciones con el entorno inmediato de los adolescentes y jóvenes fuera de su familia (Niño et al., 2017:288-290).

Siguiendo con la revisión de la literatura en el contexto mexicano, se encontró un trabajo titulado "Jóvenes en prisión: aproximaciones antropológicas en torno a la política penitenciaria”, mediante el cual se realizó un análisis del ejercicio punitivo por parte de las instituciones estatales, específicamente el sistema penal y penitenciario, dirigido a jóvenes que se hallan en reclusión; mismo que fue realizado por Vázquez Martínez (2017), y en el que reflexiona lo siguiente:

En general, las políticas públicas son comprensibles por las concepciones producidas en relación al sujeto, la sociedad y el Estado. La política penitenciaria, en particular, emplea las nociones de infracción (delito), medida (pena), tratamiento especializado y reintegración social. Sin embargo, la principal construcción social de esta política es la de comunidades para adolescentes en conflicto con la ley (penal). En esta expresión está contenida la ideología de la política penitenciaria y de las instituciones que siguen sus métodos y estrategias.

La conceptualización de adolescente a partir de la noción de individuo trae diferentes derivaciones respecto de la política penitenciaria. Por un lado, el fuerte lazo que une la concepción de adolescente con ciertas teorías psicológicas promueve la idea de un sujeto social incompleto en vías de consolidarse en la adultez mediante cualidades adquiridas en los ámbitos familiar, académico y laboral, fundamentalmente. Por otro lado, estas cualidades sólo son realizables en un contexto económico, sociocultural e histórico donde la sociedad y los vínculos sociales no se hallen fragmentados, donde haya correspondencia entre la realidad social y la política pública que actúa en esa realidad.

Se sabe que las estrategias desarrolladas por la institución penitenciaria obedecen a los principios del modelo positivista que de ninguna manera observan en la norma penal una 
construcción social con determinados intereses de grupo o de clase social, se pretende un ejercicio neutro de la fuerza punitiva respaldado con la idea de una sociedad consensual donde los valores, los medios y los fines se comparten por todos los individuos.

Si la función declarada de la política penitenciaria es la reintegración del adolescente por medio de tratamientos especializados, en realidad se castiga a los sujetos sociales por quienes son y no por los comportamientos que los condujeron a prisión. Los castigos que se practican en contra de los sujetos no tienen relación con la medida declarada, sin embargo soló son posibles a través de la política que expresa sus funciones en términos de la reintegración.

Es importante diferenciar el ámbito de análisis relacionado con los fundamentos teóricojurídicos de la función de la pena y el análisis de las prácticas sociales que se producen en el contexto carcelario, pues como pudo observarse, aun cuando ambos se hallan íntimamente relacionados en la política penitenciaria dirigida a la reintegración, el silenciamiento del sujeto no sólo se origina y reproduce mediante los documentos que los técnicos especialistas elaboran, sino mediante la negación de las prácticas violentas -actos de tortura, tratos crueles, inhumanos y degradantes- y la imposibilidad de los sujetos sociales de transformarse en autores y narradores de sí mismos y de su realidad social. De manera tal que si es viable y necesaria la investigación social dirigida a grupos vulnerados en su condición humana, habrá que comenzar por terminar con el silenciamiento que produce la política que sigue el sistema penitenciario y, en muchas ocasiones, también los propios conceptos o categorías aplicadas desde la ciencia social (Vázquez Martínez, 2017, s/p.).

Finalmente, en un trabajo titulado "Factores de riesgo asociados a la conducta violenta de los jóvenes en México”, Mancha y Ayala (2018, p. 200), llegaron a los siguientes resultados:

Que hay dos tipos de factores de riesgo que son los que más frecuentemente están correlacionados en todos los casos de conductas violentas analizados: uno tiene que ver con adicciones y el otro con comportamientos violentos en el círculo familiar o en el entorno en el que el joven se desenvuelve.

En el caso de los factores de riesgo relacionados con adicciones, se observa que los factores de riesgo encontrados consistentemente en el análisis son: en la casa del joven hay alguien que fuma, toma, se droga o apuesta; que el joven sea borracho; que el joven consuma o haya consumido drogas; que el joven tenga un amigo que toma alcohol, fuma o se droga y que en su escuela (o empleo) consuman drogas.

Los otros factores de riesgo encontrados de forma consistente en los resultados son: que en su casa lo golpeen, que tenga un amigo que golpea o traiga armas, que en su casa se empujen, griten o critiquen, tener malos maestros o jefes y vivir en una colonia clasificada como mala. Este tipo de factores nos permiten afirmar que la violencia genera violencia y que la violencia puede ser considerada como una epidemia (es decir, es contagiosa) o como un círculo viciosos que se expande (Mancha \& Ayala, 2018:200). 


\section{Conclusiones}

Derivado de la revisión de la literatura, tanto en el contexto internacional como en el mexicano, sobre lo que se ha publicado o investigado, en torno a políticas públicas para la prevención y atención de conductas atípicas en menores o jóvenes, se puede señalar que se tienen suficientes investigaciones o trabajos que pueden aprovecharse como referencia o punto de partida, para abordar esta problemática que no es ajena a México.

Sobre el tema de las políticas públicas enfocadas a la prevención y atención de conductas atípicas en menores y jóvenes, se está de acuerdo con Mancha y Ayala (2018), cuando señalan que: “Una política pública encaminada a prevenir la participación de jóvenes en actos violentos debe considerar atender de forma prioritaria el problema de adicciones (tanto al alcohol, como a cualquier tipo de droga) y tanto la adicción de los mismos jóvenes como de personas en sus círculos más cercanos (familia, amigos y escuela o trabajo)" (Mancha y Ayala, 2018: 200-201).

Sin embargo, resulta importante destacar que la problemática que enfrentan los menores o jóvenes, en cuanto a conductas atípicas, se trata de situaciones en las que intervienen diversos elementos o factores, tal vez por ello:

\section{[...], una política pública que busque prevenir} la participación de los jóvenes en actos violentos también debe enfocarse en combatir la violencia en lugares públicos (escuelas, empleos) y en las familias, con campañas y programas específicos y buscar la forma de que todo acto de conducta violenta sea denunciado públicamente y conlleve un alto costo social para quien ejerce cualquier tipo de violencia (Mancha \& Ayala, 2018:200201).

Prueba de lo anterior es que "a escala internacional se ha encontrado que programas enfocados en la familia como las escuelas para padres y los programas de escuela segura, escuela libre de violencia o educación en la cultura de la paz, tienen buenos resultados en disminuir la probabilidad de que los jóvenes se enganchen en actos violentos” (Mancha y Ayala, 2018:200201).

El autor de este documento concuerda con algunos autores que mencionan que:

[...], hay que considerar que las políticas públicas de prevención, diseñadas e implementadas a escala local, por autoridades municipales o por la misma comunidad, suelen ser más efectivas que las políticas diseñadas desde el centro del país e implementadas de forma generalizada. Asimismo, una política pública de prevención de la violencia juvenil debe ser consistente a lo largo del tiempo y para ello se requiere contar con capacidades técnicas, voluntad política, así como recursos humanos y financieros a largo plazo, ya que los resultados de una política de prevención de la violencia efectiva no son visibles en el corto plazo (Mancha \& Ayala, 2018:200-201).

\section{Referencias:}

Cienfuegos, A. G. (2015). Lo político y las políticas de la delincuencia juvenil. Una reflexión sobre el caso de jóvenes vinculados al Sistema de Responsabilidad Penal Adolescente. Bogotá-Colombia. De Prácticas y Discursos, 4(4), 1-18. 
Di Ronco, A. (2017). La estrategia europea de la prevención de la delincuencia a través del incivismo a examen. InDret, (4).

Gómez San Luis, A. H. \& Almanza A., M. (2016). Impacto del narcotráfico en jóvenes de Tamaulipas, México: drogas e inseguridad. Revista de Psicología (PUCP), 34(2), 445-472.

Llovera, M. S. (2017). Reconfiguraciones de la cuestión social: jóvenes, drogas, violencias y políticas de prevención social del delito. Publicar-En Antropología y Ciencias Sociales, (23).

Mancha Torres, G. L., \& Ayala Gaytán, E. A. (2018). Factores de riesgo asociados a la conducta violenta de los jóvenes en México. Desarrollo y Sociedad, (81), 171-210.

Montesriro, M., Samudio, T., Paternain, R., Salamano, I., Zoppolo, G., Tomasini, M., \& Henderson, J. (2016). Adolescentes, jóvenes y violencia policial en Montevideo: una aproximación descriptiva. Cuadernos de Ciencias Sociales y Políticas Sociales; 6.

Negrón C., N. L. \& García S., I. (2016). Prevención de delincuencia juvenil:¿ Qué deben tener los programas para que sean efectivos? Interamerican Journal of Psychology, 50(1), 117-127.
Nieto-Morales, C. (2012). Menores, jóvenes, educación, drogas y justicia. Barataria: revista castellanomanchega de ciencias sociales, (14), 15-28.

Niño Martínez, J. J., Osorio Ballesteros, A., \& González Ortiz, F. (2018). Prevención del delito en adolescentes en el Estado de México. La idea de riesgo y el sistema de preceptorías. Intersticios sociales, (16), 255-290.

Pérez M., N. (2016). Las políticas de prevención de la violencia y la estigmatización de los jóvenes en Michoacán (2006-2015) (Doctoral dissertation, Universidad Autónoma Metropolitana).

Vázquez Martínez, A. E. (2017). Jóvenes en prisión: aproximaciones antropológicas en torno a la política penitenciaria. Cuicuilco. Revista de ciencias antropológicas, 24(69), 229-252.

Velásquez G., R. (2009). Hacia una nueva definición del concepto “política pública”. Desafíos, 20, 149-187.

Villeda E., B. A. (2019). Influencia de las tecnologías de información y comunicación en la violencia de los niños, niñas y jóvenes en Guatemala. Revista de la Escuela de Trabajo Social de la Universidad de San Carlos de Guatemala, 1(36), 57-71. 


\section{"BASCENDERR}

Trascender, Contabilidad y Gestión Núm. 13 (enero - abril del 2020).

Universidad de Sonora. Departamento de Contabilidad.

ISSN: 2448-6388. Reserva de Derechos 04-2015-04172070800-203.

\title{
La Responsabilidad Social Empresarial Interna: Gestión Estratégica Responsable De Los Recursos Humanos Transparency of public expenditure of Sonora's Progreso Fideicomiso Promotor Urbano. 2016 fiscal year
}

\author{
Elena Martín Izquierdo ${ }^{1}$
}

Recibido: 20 de septiembre de 2019.

Aceptado: 15 de noviembre de 2019 .

DOI: https://doi.org/10.36791/tcg.v13i0.80

JEL: M14. Cultura corporativa. J53. Relaciones laborales.

\section{Resumen}

En la actualidad las personas son consideradas la fuente más importante de ventaja competitiva de las empresas, de ahí que la Responsabilidad Social Empresarial (RSE) Interna supone un enfoque que se está impregnando en las empresas, orientada tanto a sus actuales como futuros y potenciales trabajadores. Esta es considerada una inversión fundamental para su futuro, ya que una adecuada implantación y gestión de un programa de RSE, mejora el clima laboral y organizativo, aumenta el compromiso e implicación de los trabajadores, fomenta la creatividad y en general propicia un ambiente laboral agradable, donde la creatividad, la innovación, la participación y la tormenta de ideas, se convierten en características inherentes. Todo esto, hace a una empresa única e inimitable, ya que las características, habilidades y conocimientos propios creados por la interacción entre los trabajadores son únicos, convirtiéndose de este modo en ventaja competitiva.

Palabras clave: responsabilidad, social, empresarial, recursos, humanos.

\begin{abstract}
Nowadays people are considered the most important source of companies' competitive advantage, hence that Intern Corporate Social Responsibility supposes an approach which is pervading in companies, focusing on it current as well as future potential employees. It has been considered a core investment for the future, as a suitable implantation and
\end{abstract}

\footnotetext{
${ }^{1}$ Elena Martín Izquierdo. Graduada en Relaciones Laborales y Recursos Humanos. Máster Universitaria en Gestión de Personal y Práctica Laboral. Universidad de Burgos, España. Correo: emi0008@alu.ubu.es
} 
management of CSR program increases labor and organizational climate, increases employees' commitment and implication, promotes creativity and generally works for a pleasant labor climate where creativity, innovation, participation and brainstorming are changed into inherent features. All this make a company to be unique and inimitable, as features, skills and own knowledge created by employees' interaction are unique, in this way being a competitive advantage.

Keywords: responsibility, social, management, resources, human.

\section{Introducción}

Las empresas aportan valor a las sociedades a través de la creación de puestos de trabajo, la generación de estabilidad social mediante la acumulación de riqueza y el necesario apoyo en los comportamientos éticos y en los valores que deben presidir unas correctas transacciones económicas fundamentadas en la confianza. Por lo tanto, para que una organización mejore su imagen tanto exteriormente como interiormente, es necesario que su espíritu empresarial esté sostenido en un conjunto de principios alejados de la degradación de las buenas costumbres sociales.

Una segunda consideración hace referencia al material más preciado que tienen las empresas y éste no es otro que el capital humano o, más propiamente, las personas. Sin ellas, no existirían las empresas dado que estas están formadas por personas y producen y realizan actividades teniendo como horizonte la satisfacción de sus consumidores y clientes, en suma también personas. Desde la segunda mitad del siglo anterior, la consideración de los empleados más allá del mero recurso económico y material, ha dado como resultado que hoy en día, con el añadido de la batalla por el talento, se haya vuelto imprescindible tener en cuenta no sólo el bienestar general de la sociedad, en definitiva algo externo, sino también estimar la importancia y trascendencia del ámbito interno de las organizaciones, en suma, del bienestar y el clima empresariales. De ahí, la importancia de la Responsabilidad Social Empresarial Interna, por tanto este trabajo pretende analizar esta nueva orientación empresarial que engloba las cuestiones empresariales propiamente dichas, las conexiones con el entorno social y su dimensión interna.

Este trabajo es una recopilación, elaborada del modo más didáctico posible, de los aspectos que conciernen, en general, a la Responsabilidad Social Empresarial (en adelante RSE) aunque concretados en sus aspectos internos, es decir, que afectan a la denominada Responsabilidad Social Empresarial Interna (en adelante RSE Interna).

\section{Revisión de literatura}

Podemos encontrar diferentes definiciones del concepto de Responsabilidad Social Corporativa, entre las que se pueden destacar la propuesta en el Libro Verde "Fomentar un marco europeo para la responsabilidad social de las empresas" que fue publicado por la Comisión Europea en 2001, como consecuencia del mandato realizado en el Consejo Europeo de Lisboa celebrado en marzo del 2000, donde se indica que, se trata esencialmente de "un concepto con arreglo al cual las empresas deciden voluntariamente contribuir al logro de una sociedad mejor y un medio ambiente más limpio" y se define como "la integración voluntaria por parte de las empresas, de las preocupaciones sociales y medioambientales en sus operaciones comerciales y sus relaciones con sus interlocutores"1.

\footnotetext{
${ }^{1}$ En el mismo Libro Verde, se indica que el porvenir de la Unión Europea está en alcanzar un nivel suficiente de bienestar social y de progreso para todos sus ciudadanos.
} 
El concepto de Responsabilidad Social se relaciona con la parte voluntaria de la actividad de las empresas que tiene que ver con la mejora de la sociedad de la que forman parte y con la preocupación por el medio ambiente en el que se encuentran.

Un mayor número de empresas, cada vez más, reconocen abiertamente su responsabilidad social y la consideran parte de su identidad como nos indican Server y Villalonga (2005). Esta responsabilidad la deben comunicar frente a sus propios trabajadores $\mathrm{y}$, en general, ante todos los stakeholders de la empresa que pueden influir en su éxito.

La preocupación acerca de estos temas de índole social y medioambiental, es la que genera una demanda de información que a su vez desemboca en la necesidad de disponer de un documento en el que se recoja dicha información demandada por la sociedad y que debe ser ofrecida por las empresa.

\section{Objetivos}

El objetivo general de este trabajo es conocer, las actividades de Responsabilidad Social Empresarial Interna (RSE Interna), analizando específicamente las cuestiones relativas al nivel interno de las empresa, todo ello partiendo de un mercando globalizado e internacionalizado que afecta tanto a su actividad, como a las políticas adoptadas para afrontar la misma.

De este objetivo general se desprenden los siguientes objetivos específicos:

a) Delimitar la RSE Interna y su orientación en el marco de la Estrategia y la Política aplicadas a los recursos humanos de la empresa.

b) Determinar los ámbitos de actuación de la RSE Interna que afectan al conjunto de la organización. c) Conectar la estrategia de recursos humanos, con el planteamiento sobre la RSE Interna, como con cada uno de los procesos esenciales que la conforman.

d) Especificar los elementos que componen la RSE Interna.

e) Conectar, la RSE Interna, con las nuevas tendencias sociales relacionadas con la gestión en la organización mediante su involucración y participación en los procesos de Recursos Humanos.

f) Establecer un plan de actuación en materia de RSE Interna para la empresa.

g) Reflexionar sobre la RSE Interna, y concienciarse de la importancia de esta tendencia como elemento de atracción sobre la empresa, tanto para la posibilidad de nuevas incorporaciones, así como, de maniobra para el fomento de la permanencia.

Debido a que la responsabilidad social, tanto en su ámbito externo como interno, ha ganado y está ganando importancia a nivel internacional, ya que a pesar de que se trate de un recurso intangible, se convierte en un activo trascendente en una organización. Lo que pretendo exponer a continuación, es como su aplicación resulta beneficiosa en todas las empresas y particularmente en aquellas integradas en la producción y el comercio globalizado.

\section{Metodología}

La metodología empleada, fue una investigación documental:

- En primer lugar, se acude a la interpretación realizada por algunos analistas, de los cambios y movimientos sociales. 
- En segundo lugar, se han examinado libros, guías, monografías y artículos científicos más relevantes en la materia.

- En tercer lugar, se ha completado el estudio con datos procedentes de las empresas más representativos.

En último lugar, se han analizado los contenidos, para poder generar así inferencias contextuales.

\section{Resultados obtenidos}

En la actualidad, las personas son consideradas la fuente más importante de ventaja competitiva de las empresas y, por lo tanto, se gestión ha dejado de ser percibida como un coste para ser considerada una inversión fundamental para su futuro. Un estudio realizado por SAM y el Boston Consulting Group en 2003 concluyo que existe una correlación positiva entre la gestión del capital humano y el rendimiento financiero. Estas compañías tienen éxito a la hora de atraer y retener a los mejores empleados, y saben anticipar sus expectativas cambiantes en relación a temas como los contenidos de los puestos de trabajo, la flexibilidad en la forma de trabajar, el disfrute de beneficios sociales adicionales, la formación y el desarrollo profesional (Sustainability, 2005).

Tomando como referencia la parte del Libro Verde dedicada a la RSE Interna, supone considerar que las prácticas responsables en lo social interior afectaban a cuatros áreas de actividad: la Gestión de los Recursos Humanos, la Salud y la Seguridad en el lugar de trabajo, la Adaptación al cambio ${ }^{1}$, la Gestión del Impacto Ambiental y de los Recursos Naturales ${ }^{2}$.
Los objetivos de una gestión responsable de los recursos humanos son (Navarro, 2012):

a) Conseguir el alineamiento de los integrantes de la organización con la visión, la misión y los valores organizativos.

b) Lograr el máximo compromiso e involucración de los mismos en la consecución de los objetivos de la empresa.

Los temas fundamentales que las empresas han de considerar y promover en su gestión de los recursos humanos para ser una empresa responsable y sostenible son los siguientes (Carneiro, 2008):

1) Las Responsabilidades de la Organización: las cuales afectan a la incorporación de la sensibilidad sobre las cuestiones sociales y del cuidado de su aplicación interna a los aspectos sustanciales y estratégicos de una empresa, llegando incluso a favorecer una cultura de empresa orientada a lo social.

2) La Responsabilidad sobre las Personas: es decir, yendo más allá de las tradicionales intervenciones sobre el clima laboral y personal, la RSE Interna plantea otras visiones así como una nueva orientación sobre lo relativo al desarrollo profesional y humano de los integrantes de las empresas.

3) La Responsabilidad sobre las Relaciones: lo que está vinculado a esta disposición de servicio al cliente interno que la RSE Interna.

\footnotetext{
${ }^{1}$ Centrándonos en el ámbito estratégico, específicamente en la denominada orientación general, dado que es un reto complejo, ya que procede del exterior, y además, exige una reacción por parte de toda la empresa. Y es ahí donde el departamento de Recursos Humanos puede tomar la bandera de su orientación y estar absolutamente involucrada en su resolución. Por supuesto, la RSE Interna también puede colaborar como forma de actuación de manera directa.

${ }^{2}$ Se tratará desde la orientación de la RSE Interna.
} 
4) La Responsabilidad sobre el Ambiente ${ }^{1}$ : hace referencia a ámbitos como la ecología interna o la aplicación de medidas de mejora social medioambiental que incluso pueden tener un traslado al conjunto de la sociedad.

Un aspecto práctico, para poder implementar y obtener los beneficios de una gestión responsable de los Recursos Humanos, es través de la implementación, de un Plan de Acción, orientado hacia esos objetivos, que consiste en una línea organizada de acciones de RSE Interna, que permitan la implantación de dichas acciones de RSE, tanto en una gran empresa como una pyme. El modelo propuesto constará de 5 pasos y 13 acciones (Guía de Responsabilidad Empresarial Interna y Recursos Humano, 2008).

El Plan Estratégico para una implantación paulatina, coordinada y adecuada de la RSE Interna en las empresas deberá seguir estos pasos (Carneiro, 2008):

1. Planteamiento Estratégico. Se trata de la conexión tanto con el Plan Estratégico de la Organización, como con sus grandes líneas de actuación ${ }^{2}$. Es una función que suele llevar a cabo la dirección, que se funda en la sensibilidad hacia las cuestiones desarrolladas en el capítulo anterior, y en el convencimiento y la involucración.

2. Situación de partida. Se realizan los planteamientos generales sobre la RSE que tenga la compañía de una manera realista. De esta manera, es necesario seguir los siguientes puntos: a) Planteamientos estratégicos sobre la Responsabilidad Social: se refiere, a lo que realice la empresa en dicha materia (No hay que olvidar que la RSE Interna es una parte más de la RSE).

b) Auditoria de lo realizado en temas sociales: está relacionado con la RSE en general, pero que afecta en mayor grado a su aspecto interno. Saber qué se está haciendo en estas cuestiones así como sí existen programas de actuación social e incluso si hay personas dentro de la empresa que son partícipes de actividades de tipo social ayuda a orientar las actuaciones en materia de RSE Interna. Pero, sobre todo, evita duplicidades innecesarias ${ }^{3}$.

c) Determinación de la estructura previa necesaria: es necesario ubicarla en el organigrama o al menos designar la persona o personas responsables de la RSE Interna ${ }^{4}$.

d) Conexión con otras áreas de la empresa: es razonable insertarla en función de Recursos Humanos, dado el carácter de los temas tratado dentro de la RSE Interna. Puede suponer para la función un nuevo punto de vista y por tanto adherirse a lo que ya se esté haciendo en materia de gestión de personas o bien puede nombrarse a alguien del departamento para que desarrolle todo lo relacionado con la RSE Interna.

\footnotetext{
${ }^{1}$ Se recogido en el Libro Verde de la Comisión Europea aunque con una clara orientación externa, pero permitiendo de este modo que la evolución de la RSE Interna comience a introducirse en este ámbito.

${ }^{2}$ Estén estas o no debidamente recogidas en una base documental.

${ }^{3}$ La auditoría es previa al establecimiento de los recursos necesarios debido a que hay empresas que no pueden soportar tener una estructura específica para estas cuestiones y por ello las ponen en departamentos ya existentes.

${ }^{4}$ En la mayoría de los casos suele alojarse en el departamento de Recursos Humanos, pero puede depender también de algún área propiamente de RSE e incluso de Comunicación Interna o en relación directa con Presidencia o con el Consejo de Administración. Existen ya empresas que han empezado a crear departamentos específicos para tratar las cuestiones que afectan a la RSE Interna., en el caso de ser mínima la estructura de gestión de las personas supone la asimilación de la orientación.
} 
3. Modelo de RSE Interna propio. En este momento, hay que plantear las grandes líneas de acción en materia de la organización, las personas, las relaciones y el ambiente. En definitiva, es especificar, a grandes rasgos, lo que resulta posible llevarse a cabo.

Además de esto, resulta importante abordar lo siguiente:

a) Generar la imagen deseada a través de la puesta en marcha de actuaciones tanto en lo que corresponde a la RSE en general como a la Interna. Y ello se debe a que no todas las empresas tienen el mismo tipo de enfoque sobre su presencia en la sociedad ${ }^{1}$.

b) Debido a esta situación, resulta de interés revisar la definición de los valores y los mensajes a transmitir por parte de la empresa en su relación tanto con el entorno externo como hacia su ecosistema interno.

4. Operativa. Hace referencia a la puesta en marcha de lo previamente diseñado. Cinco son las cuestiones a tratar:

1) Diseño de un plan de comunicación interno y externo. Habiendo decidido ya si existe interés en dar a conocer los logros en materia de RSE o de RSE Interna, estos deben conectarse con lo que en cuestiones de notoriedad y comunicación que realice la empresa ${ }^{2}$.

2) Diseño de un plan de comunicación de la función: Significa que, sobre todo internamente, es necesario dar a conocer la orientación que desde, por ejemplo, Recursos Humanos se ha tomado; pero también qué tipos de acciones se van a poner en marcha en esa dirección ${ }^{3}$.

3) Lanzamiento e implantación de la orientación que supone la RSE Interna: sobre todo para la gestión de las personas.

4) Movilización del Capital Humano de la organización: a través de diversos medios como pueden ser las reuniones informativas, el uso de herramientas de comunicación interna, los periódicos de empresa, los portales de empleado o una mera notificación.

5) Análisis de los resultados de la puesta en marcha de la orientación: es un paso imprescindible que se da en cualquier diseño de un plan de actuación en el interior de una empresa.

5. Planificación y Acciones. Supone ir más allá de la mera implantación. Agrupa:

- El diagnóstico de la situación, es decir el control constante del progreso de la iniciativa.

- $\quad$ La elaboración de proceso.

- El diseño del plan de comunicación de lo que se vaya consiguiendo

- El conjunto de las acciones operativas.

\footnotetext{
${ }^{1}$ Algunas empresas no quieren dar publicidad a aquello que realizan en materia de RSE, incluso con respecto a la interna; otras, por el contrario, dan publicidad a cualquier actuación que pueda tener un traslado hacia la sociedad. En todo ello influirán cuestiones como la obligación que deriva de las certificaciones, la presentación obligada de información social, tal es el caso de las empresas cotizadas en bolsa y las Cajas de Ahorros, o el control que sobre ellas ejerzan los stakeholders.

${ }^{2}$ Es el caso, de las memorias de RSE o en las de Sostenibilidad, o, cuando ocurran algunas acciones muy puntuales y de repercusión en el entorno inmediato, su aparición en medios de comunicación. Además, Dado el carácter sensible que todas estas cuestiones tienen en las relaciones entre las empresas y el entorno, es muy recomendable su control y tratamiento profesional.

${ }^{3}$ De no ser así, el grado de aceptación y participación por parte de los empleados será mucho menor o, incluso, inexistente.
} 
“En definitiva, corresponde a la implementación de lo propuesto en los cuatro pasos anteriores y suelen ir en paralelo a lo expuesto a partir del segundo punto, dado que, en numerosas ocasiones y dada la situación en la que las empresas se encuentran, no se puede sólo diseñar sin implantar. Un modo realista de acercarnos a estos planteamientos nos sugiere que tiene que ser realizado todo en paralelo, esto es, diseño, comunicación, gestión, implantación y control, pero sin olvidar los pasos propuestos que se han de dar, aunque estos, en muchas ocasiones, se solapen” (Carneiro, 2008:129).

\section{Conclusiones}

Del Trabajo realizado cabe extraer las siguientes conclusiones:

1. La RSE se ha convertido en algo más que una mera moda; supone un enfoque que se está impregnando en las empresas, fruto de una demanda tanto externa como interna y que resulta inevitable para las empresas.

2. En la actualidad las personas son consideradas la fuente más importante de ventaja competitiva de las empresas y, por lo tanto, su gestión ha dejado de ser percibida como un coste para ser considerada una inversión fundamental para su futuro.

3. La RSE Interna es una novedosa orientación, un punto de vista distinto, en línea con la RSE en general, que consiste, básicamente, en asumir la realidad social en general, la demanda comunitaria sobre las empresas, la sensibilidad social organizativa y la propiamente laboral, e insertarla en el corazón de la gestión empresarial de las personas.

4. La RSE Interna es sensible a las situaciones personales y concretas de los empleados que afectan a la mejora en el clima y en el ambiente empresarial y organizativo.

5. Los objetivos fundamentales de la gestión responsable de los recursos humanos son conseguir el alineamiento de los integrantes de la organización con la visión, la misión y los valores organizativos, y lograr el máximo compromiso e involucración de los mismos en la consecución de los objetivos de la empresa.

6. Aunque el departamento de recursos humanos tiene una responsabilidad incuestionable en la gestión de los temas mencionados, sería una equivocación considerar que la gestión responsable de los recursos humanos atañe exclusivamente o fundamentalmente a dicho departamento. Por el contrario, la responsabilidad de dicha gestión ha de ser asumida por todos los directivos y mandos de la organización.

7. Las empresas han de estar siempre atentas a la coherencia entre sus sistemas de gestión y cualquiera de sus iniciativas.

8. La RSE Interna, tiene la obligación de seguir normativas concretas; así lo demuestran iniciativas como la poco conocida y extendida, al menos en España, norma "Investors in People", Inversores en personas, que trata de organizar la formación y el desarrollo del personal en clave de procesos y procedimientos certificables.

9. La RSE Interna puede cumplir un papel orientador, de carácter trasversal a todos los procesos de recursos humanos y con una orientación en cuatro vertientes: la correspondiente al aspecto organizativo, compuesta por la gestión del cambio organizativo, la mecánica de las relaciones laborales y todo lo referente a la Salud y 
Seguridad en el trabajo; la dimensión de las personas, que agrupa lo referente al talento, a las políticas de igualdad y de no discriminación y a la gestión de los colectivos especiales, en particular a lo que corresponde a los inmigrantes; una tercera faceta que corresponde a la creación de relaciones, en concreto a la eliminación de los riesgos psicosociales en el trabajo, el fomento de la participación y a la implantación de la acción social a través, fundamentalmente, de los programas de voluntariado corporativo; y en cuarto y último lugar, aquello que afecta a la creación y mantenimiento del ambiente, que corresponde tanto a la ecología organizativa interna, como a la conciliación laboral con la personal y familiar, con una especial mención a lo que afecta a la mejora en la gestión del tiempo y de los horarios, y, en último lugar, al incentivo interno con traslado hacia el exterior de la gestión medioambiental.

10. Para mejorar las actitudes y aptitudes creativas de sus personas, las empresas deben tomarse muy en serio el establecer las condiciones necesarias para que la creatividad florezca y ofrecer formación en técnicas de creatividad.

11. Las metodologías de formación tradicionales tienen un escaso o nulo impacto en la mejora de las habilidades emocionales, así como en el cambio de valores. Sin embargo, se ha demostrado que el uso de técnicas introspectivas y mediativas tienen un alto impacto en ambos temas.

12. La inteligencia emocional marca la diferencia en el rendimiento profesional de las personas en una medida mucho mayor que el coeficiente intelectual.
13. En la nueva economía de la empresa responsable y sostenible los empleados han de contribuir, además de con su tiempo y esfuerzo, con todo su potencial. Las empresas han de conseguir el continuo desarrollo de los conocimientos y experiencias, actitudes y aptitudes de cada uno de sus integrantes. Asimismo, han de conseguir que el conocimiento de la organización en su conjunto supere la mera suma de los individuales. Ya que, al no ser imitable, el conocimiento organizativo que reside en una pluralidad de personas y equipos es una fuente sostenible de ventaja competitiva.

14. Por ultimo señalar, que a las empresas les afecta todo lo humano, ya que estas son un entramado de relaciones, tanto internas como externas. Por tanto, esta incentivada de orientación social les exige, a ellas, a sus dirigentes y en concreto al departamento de gestión de personas correspondiente, realizar la debida respuesta coordinada y organizada. Y la RSE Interna resulta ser ese tipo de respuesta.

Teniendo presentes todas las conclusiones anteriormente descritas, podemos llegar a una reflexión final y es que si las empresas quieren retener y atraer a los mejores talentos, tendrán que ajustarse y adaptarse a las tendencias del momento, por lo tanto tienen que realizar transformaciones en ellas.

\section{Referencias:}

Álfaro, T. (2014). Retención del talento. Madrid. España: Editorial Empresarial S.L.

Alvarado, A. y Walleska, M. (2008). Dimensionalidad de la responsabilidad social empresarial percibida y sus efectos sobre la imagen y la reputación. Estudios Gerenciales, 24(108), 37-59. 
Amabile, T. (1998). How to Kill creativity. Harvard Business Review, 76,44-67.

Anca, C. y Vázquez, A. (2005). La gestión de la diversidad en la organización global. Madrid, España: Editorial Person.

Carneiro, M. (2008). Guía de Responsabilidad Social Empresarial Interna y Recursos Humanos. Recuperado de http://www.bibliotecavirtual.info/wpcontent/uploads/2012/02/guia_rse_interna_y_recursos_ humanos.pdf

Carneiro, M. (2004). La Responsabilidad Social Corporativa Interna. La "nueva frontera" de los Recursos Humanos. Madrid, España: Editorial ESIC.

Comisión Europea. (2001). Libro Verde: Fomentar un marco europeo para la responsabilidad social de las empresas. Comisión de las Comunidades Europeas. Recuperado de http://eur-lex.europa.eu/legalcontent/ES/TXT/PDF/?uri=CELEX:52001DC0366\&fr om $=\mathrm{ES}$

Correl, J. (2007). Dirección de personas: escuchar, influenciar y desarrollar a sus colaboradores. Madrid, España: Editorial Netbiblo, colección Business Pocket.

Durán, G. (2007). Empresas y Medio Ambiente: Políticas de Gestión Ambiental. Madrid, España: Editorial Pirámide.

Fernández, M. (2007). Motivar con la acción social. A Coruña, España: Editorial Netbiblo, colección Business Pocket.

Frances, P., Borrego, A. y Velayos, C. (2003). Códigos éticos en los negocios: creación y aplicación en empresas e instituciones. Madrid, España: Editorial Pirámide.
García, J.L. (2 de septiembre de 2013). Cómo atraer, motivar y retener el talento en las organizaciones. Gestionando personas. Recuperado de http://www.gestionandopersonas.com/como-atraermotivar-y-retener-el-talento-en-las-organizaciones/

Godfrey, P., Merrill, C. y Hansen, J. (2 de Diciembre de 2008). The relationship between corporate social responsibility and shareholder value: an empirical test of the risk management hypothesis. Strategic Management Journal. 30: 425-445. doi: 10.1002/smj.750.

Herrero, C. y Javier, F. (2007). Igualdad y empresa. Madrid, España: Editorial Pearson Prentice Hall.

Hillier, C. y Agilar, J. (2006). En busca del compromiso. Madrid, España: Editorial Almuzara.

Jericó, P. (2007). Gestión del talento. Madrid, España: Editorial Prentice Hall.

Lindsay, G. (2009). Ética profesional y psicología. Papeles del Psicólogo, 30(3), 184-194. Recuperado de http://www.papelesdelpsicologo.es/pdf/1747.pdf

López, J. (2005). La ecología social de la organización. Madrid, España: Editorial La Muralla.

Nonaka, I. y Takeuchi, H. (1995). The Knowledge Creating Company. Londres, UK. Oxford University Press.

Saldarriaga, J.G. (2013). Responsabilidad social y gestión del conocimiento como estrategias de gestión humana. Estudios Gerenciales, 20, 110-117.

Surroca, J., Tribó, J. y Waddok, S. (28 de septiembre de 2009). Corporate Responsibility and financial performance: the role of intangible resources. Strategic Management Journal. 31: 463-490. doi: 10.1002/smj.820. 
Villalonga, I. y Server, R.J. (2005). La Responsabilidad Social Corporativa (RSC) y su gestión integrada. CIRIEC, 53, 137-161.

Wang, S. (2004). Staged financing in venture capital: moral hazard and risks. Journal of Corporate Finance, 10(1), 131-155. doi: 10.1016/S0929-1199(02)00045-7

Witt, P. (8 de Julio de 2014). Responsabilidad social del departamento de recursos humanos. LosRecursosHumanos.com. Recuperado de http://www.losrecursoshumanos.com/contenidos/5456responsabilidad-social-del-departamento-de-recursoshumanos.html

Zabala, I. (2008). La dimensión Interior. Madrid, España: Editorial Pirámide. 